Methods A quasi-experimental study of the clinical effectiveness of a novel risk-driven digital outreach program in two distinct Medicaid cohorts: Children with asthma and Pregnant women at risk for preterm birth vs. matched controls.

Results Among Medicaid children with asthma, program participation is associated with $24 \%$ increase in outpatient visits, $24 \%$ reductions in emergency department visits, and $15 \%$ increase in controller medication use. Among Medicaid pregnant women, program participation is associated with $24 \%$ increase in prenatal visit attendance, $27 \%$ reductions in early preterm deliveries ( $<35$ weeks), and 54\% reductions in baby costs in the first year of life. Overall Program Satisfaction is high.

Conclusions Targeted text messaging tailored to patient risk profiles drives healthy behavior changes and improved health

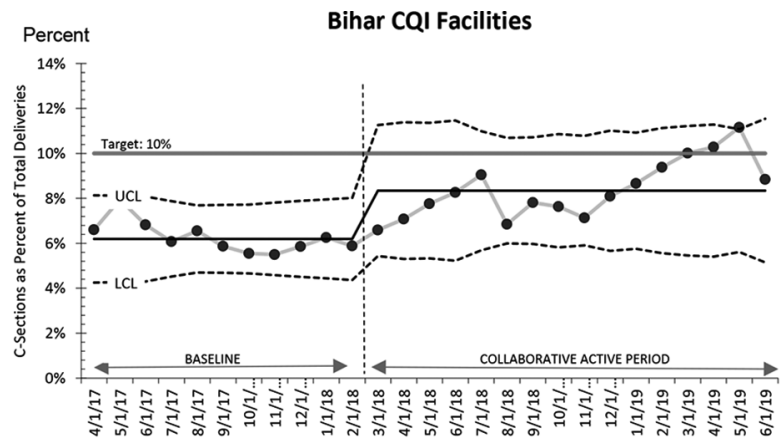

Abstract 14 Figure 1 Prevalence of C-section deliveries

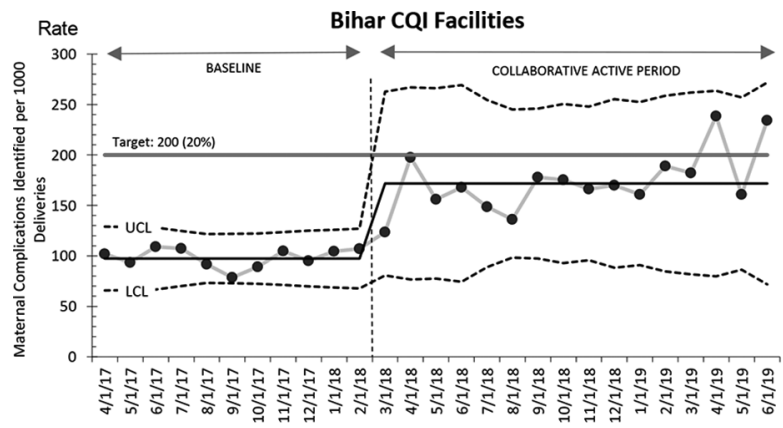

Abstract 14 Figure 2 Rate of identification of maternal complications

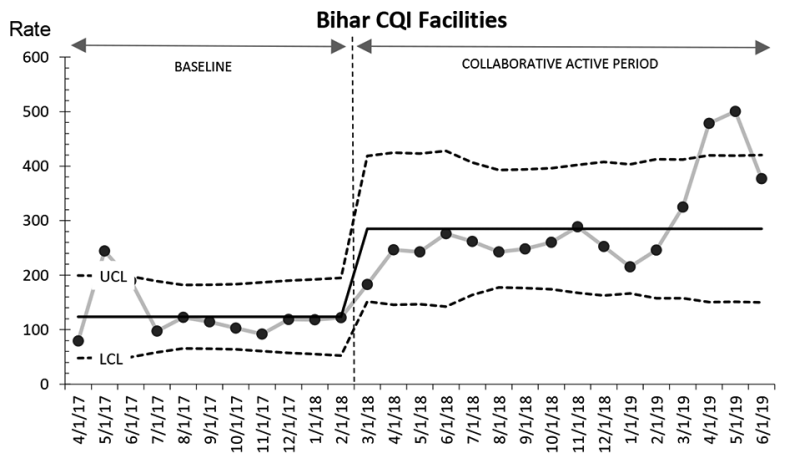

outcomes in diverse health cohorts. Leveraging advanced analytics to drive personalized digital health engagement is essential for success.

\section{A QUALITY IMPROVEMENT COLLABORATIVE TO IMPROVE MATERNAL COMPLICATION IDENTIFICATION AND USE OF C-SECTION AND BLOOD TRANSFUSION IN BIHAR, INDIA}

${ }^{1}$ Clark Jackson, ${ }^{1}$ Abha Mehndiratta, ${ }^{1}$ Gareth Parry, ${ }^{2}$ Hemant Shah, ${ }^{2}$ Tanmay Mahapatra, 2Prabir Moharana, 'Pierre Barker, 'Azhar Ali. ${ }^{1} / H I$, USA; ${ }^{2}$ CARE India, India

10.1136/bmjoq-2019-ihi. 14
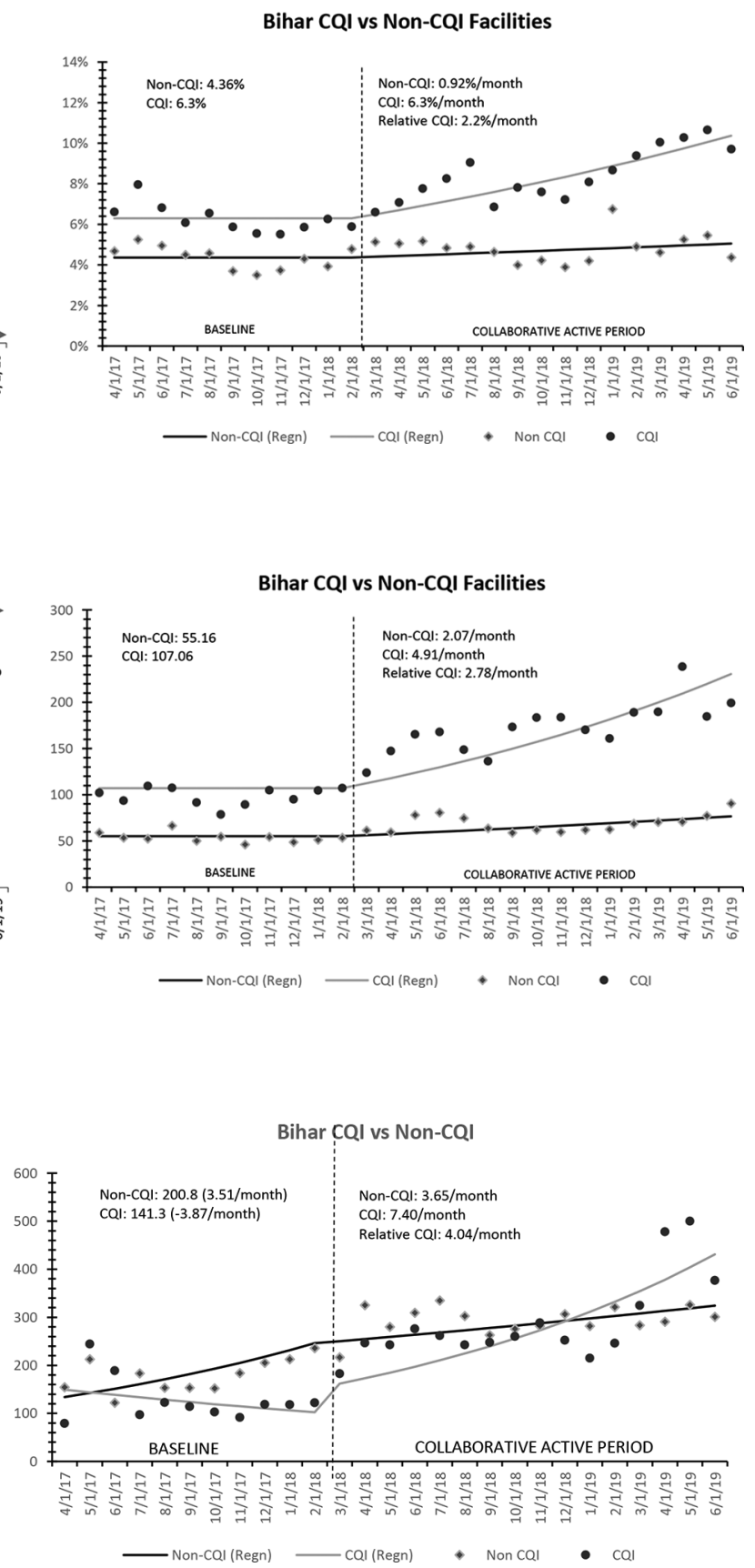

Abstract 14 Figure 3 Rate of complicated pregnancies treated with blood transfusion (per 10,000 institutional deliveries) 
Background Bihar, India has higher maternal mortality rates than the country on average. A Collaborative Quality Improvement (CQI) program was created to improve data transparency and accuracy, with the aim of increasing maternal complication identification and improving key life-saving processes for cesarean section and blood transfusion.

Objectives To estimate the impact of the CQI program on identification of maternal complications and use of C-section and blood transfusion for maternal complications in participating hospitals relative to non-participating facilities in Bihar.

Methods Ten district hospitals participated in the collaborative from February 2018 to September 2019. Activities included leadership and improvement workshops; learning sessions; and monthly onsite support provided by IHI and CARE India. Data from HMIS was used to assess change from baseline to after the start of the collaborative in CQI district hospitals, compared to non-participating district hospitals for: maternal complication identification, C-sections, and blood transfusions.

Results Figures 1-3 illustrate improvement over time for all measures. For example, across all CQI hospitals, control chart methodology suggested C-sections (percent all births) increased from a rate of $6.2 \%(95 \%$ CI $5.9 \%, 6.7 \%)$ at baseline to $8.3 \%(95 \%$ CI $7.8 \%, 9.0 \%)$ after the start of the collaborative. Comparative analysis found $\mathrm{C}$-sections increased by $2.2 \%(95 \%$ CI $1.8 \%, 2.64 \%)$ per month in CQI facilities during the collaborative period, relative to non-CQI facilities.

Conclusions Application of this QI collaborative led to improvement in identification of maternal complications and use of C-section and blood transfusion.

\section{AN INNOVATIVE INTERVENTION TO IMPROVE RESPECTFUL MATERNITY CARE IN THREE DISTRICTS IN ETHIOPIA}

${ }^{1}$ Birkety Jembere, ${ }^{1}$ Haregeweyni Alemu, ${ }^{2}$ Munir Kassa, ${ }^{3}$ Kedest Mathewos, ${ }^{1}$ Mehiret Abate, ${ }^{1}$ Befikadu Bitewulign, ${ }^{1}$ Kendra Njkou, ${ }^{2}$ Meseret Zelalem, ${ }^{3}$ Neil Prose, ${ }^{1}$ Hema Magge. ${ }^{1} \mathrm{HI}$, Ethiopia; ${ }^{2}$ FMOH, Ethiopia; ${ }^{3}$ Duke Global Health Institute, USA

\subsection{6/bmjoq-2019-ihi.15}

Background Disrespect and abuse (D\&A) during childbirth are major violations of human rights and often deter women from accessing skilled delivery. In Ethiopia, D\&A has been documented to occur in $49.4 \%$ of mothers delivering in health facilities.

Objectives To describe the development, implementation and results of a novel intervention to improve Respectful Maternity Care (RMC) in three districts in Ethiopia.

Methods As part of a national initiative to reduce maternal and perinatal mortality in Ethiopia, we developed a novel RMC training module with three core components; testimonial videos, didactic session on communication, and onsite coaching to devise local solutions that enhance RMC. We implemented these in three districts in each of Oromia; Southern Nations, Nationalities, and Peoples' and Tigray regions as of February 2017. We deployed mixed methods to assess effectiveness of the approach. Measures of births with privacy and birth companion from a 27 month data from 20 health facilities were analyzed using STATA version 13 for interrupted time series. Facilitated discussion with health care providers was held to assess feedback. 